\title{
Extensive persistent Trichuriasis: A case report
}

\section{Supriya B. Gachinmath, Pratibha S., Mary Dias, P. Mallikarjun ${ }^{1}$}

Department of Microbiology, ${ }^{1}$ Gastroenterology, St. John's Medical College and Hospital,

Bangalore, Karnataka, India

Address for the Correspondence: Dr. Supriya BG,

Department of Microbiology, St. Johns Medical College, John Nagar, Sarjapur Road, Bangalore,

Karnataka - 560 034, India.

E-mail: supriya_bg@rediffmail.com

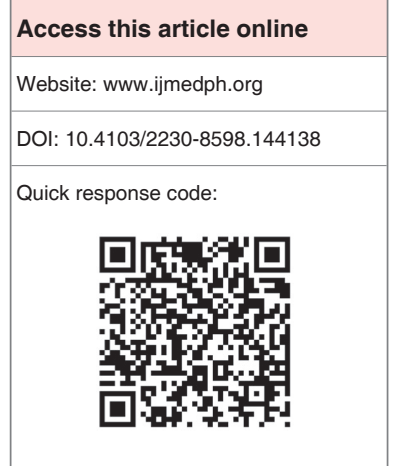

Trichuris trichura (whip worm) is one of the most common intestinal parasite of humans in tropical countries. Often asymptomatic in humans, heavy infection can lead to chronic bloody diarrhoea associated with complications like rectal prolapse (particularly in children), appendicitis, colitis and proctitis. Very few cases of extensive Trichuriasis have been reported worldwide.

We report a case of a 5-year-old girl who presented with generalized weakness, bleeding per rectum and rectal prolapse of 1 year duration. She was investigated for bleeding per rectum. Colonoscopy performed revealed extensive worm infestation involving the entire colon which was later confirmed as Trichuris trichura (whip worm) by microscopy. Child was diagnosed as Trichuris Dysentery Syndrome (TDS), treated for the same and discharged on improvement of the symptoms. The patient was traced back using the address available from the hospital records as she was not brought to the hospital for further follow up. Although none of her symptoms recurred, her stool microscopy however showed the presence of Trichuris trichura ova even at the end of 2 years indicative of persistent asymptomatic Trichuris trichura infection.

Key words: Colonoscopy, Trichuris Dysentery syndrome (TDS), Trichuris trichura

\section{INTRODUCTION}

Trichuris trichura is a soil transmitted intestinal helminth prevalent in warm, moist, tropical and subtropical countries with poor sanitation. Prevalence is especially high in children reaching up to $95 \%$ in these regions. ${ }^{[1]}$

Though most Trichuris trichura infections remain asymptomatic, a minority who have heavy parasitic infection become symptomatic which include anemia, diarrhoea and intestinal bleeding. ${ }^{[2]}$ Rectal prolapse due to Trichuris trichura infection is also rarely reported in patients with heavy parasite load.

The simplest and easiest method of diagnosis of Trichuris trichura infection is based on the demonstration of characteristic bile stained barrel shaped egg in the stool, however in some cases colonoscopy and computerized tomography are found to be helpful especially when they are being investigated for symptoms like intestinal bleed and rectal prolapse. ${ }^{[3]}$

Here we report a case of extensive and persistent chronic Trichuriasis in a child presenting with symptoms of anemia, bloody diarrhoea and rectal prolapse.

\section{CASE REPORT}

A 5-year-old female child hailing from an urban slum visited the paediatric out-patient department presenting with complaints of rectal bleeding and fatigue of 1 year duration. On general physical examination the remarkable findings were that of marked pallor and mild rectal mucosal prolapse. Her investigations revealed hemoglobin of $2.4 \mathrm{~g} \%$, total leukocytes count of 19,700 cells $\left(\mathrm{N}_{41} \mathrm{~L}_{47} \mathrm{E}_{11}\right)$, and platelet count 3.7 lakhs. Peripheral smear showed microcytic hypochromic blood picture suggestive of iron deficiency anemia with leukocytosis and eosinophilia. Stool was positive for presence of occult blood.

Stool microscopy with saline preparation revealed numerous RBCs/HPF and ova of Trichuris trichura that were bile stained, barrel shaped with mucous plugs on either end [Figure 1]. Colonoscopy 
undertaken for the evaluation of lower gastrointestinal bleed, revealed extensive worm infestation involving entire colon and terminal ileum [Figures 2a and b].

The child was treated with serial packed RBCs transfusions, following which her hemoglobin status improved to $10 \mathrm{~g} \%$. She was also treated with Syp. Mebendazole $5 \mathrm{ml}$ twice daily for 3 days. Iron supplements were started which she was asked to continue for 3 months.

Child showed significant improvement of anemia and complete cessation of bleeding per rectum before discharge from hospital. She was advised to come for review after 2 weeks. However she was not brought back for further follow up. The child was then traced back using her medical records at the end of 2 years. She had remained asymptomatic during this time and therefore her parents did not consider the necessity for further follow up at the hospital. Stool sample obtained at that point remained positive for ova of Trichuris trichura by microscopy.

\section{DISCUSSION}

Trichuris trichura is one of the most common human parasites to be found worldwide, particularly in countries with warm humid climates. Overall prevalence of whipworm infestation is up to $90 \%$ in rural areas of tropical and subtropical countries including South East Asia, Africa, Central and South America with poor hygiene and low level of awareness among the lower socio-economic population. ${ }^{[4]}$

Though Trichuriasis is one of the commonest soil transmitted helminthiasis worldwide, most children remain asymptomatic by harboring only small number of worms and do not proceed to severe infection. In massive infection, patients present with Trichuris trichura dysentery syndrome consisting of abdominal pain, tenesmus, and bloody diarrhoea with rectal prolapse which was also seen in our case. ${ }^{[5]}$

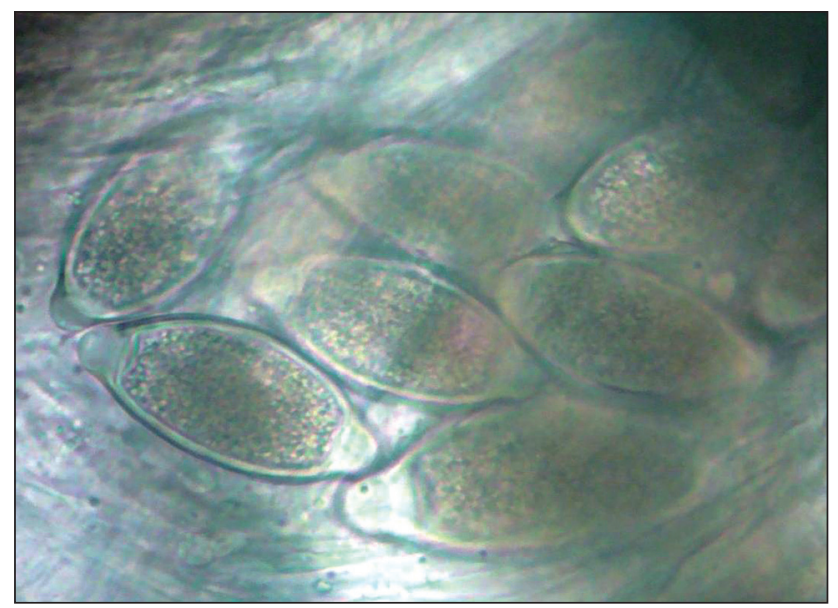

Figure 1: Microscopic picture of multiple ova observed in the lumen of the Whipworm $(\times 400)$
This child came from an urban slum area belonging to a joint family with multiple siblings. The cause of her bleeding per rectum and rectal prolapse was traced back to extensive Trichuris trichura infestation that was conclusive only by way of colonoscopy. Several reports support our finding of Colonoscopy being an important tool for diagnosing Trichuriasis infection along with stool microscopy. ${ }^{[5,8,9]}$

Kyung Sun Ok et al. ${ }^{[9]}$ reported four cases of Trichuriasis infection which were diagnosed by detection of the parasite by colonoscopy. Two of four cases showed barrel shaped bile stained eggs with mucoid plugs at both the ends in stool microscopic examination.

Other two cases presenting with abdominal pain and tenesmus were negative for parasitic eggs in stool examination but their colonoscopy revealed a pearly white small worm which was removed from the ascending part of the colon. On microscopic examination, it was confirmed to be a male Trichuris trichura parasite. Since it was a single male whipworm, stool examination for parasitic egg was negative. These results suggest that colonoscopy is a useful diagnostic approach for parasitic infections even for asymptomatic patients and for patients with negative stool examination. ${ }^{[8,9]}$

Similar cases have been reported by Daniel et al., where in Trichuris trichura presented with chronic dysentery like syndrome which can mimic other inflammatory bowel disease like celiac and ulcerative colitis. ${ }^{[5]}$

According to Stephen et al., ${ }^{[7]}$ the Trichuris Dysentery Syndrome associated with heavy Trichuris trichura, manifest with chronic dysentery, rectal prolapse, anemia, poor growth and clubbing of the fingers which is commonly seen in children. This profound growth stunting in TDS can be reversed by regular periodic treatment for the infection and initial oral iron supplement. As per WHO Essential drug list, Mebendazole and Albendazole are very effective against Trichuris trichura, multiple doses are required to attain complete parasitological cure in these cases. ${ }^{[7]}$

Since our patient did not come back for further follow up, she did not receive periodic deworming which is recommended in such cases to prevent reinfection or relapse of symptoms. ${ }^{[6]}$ Further the other members of the family need screening and periodic deworming as they may probably act as the source of her persistent infection. Also

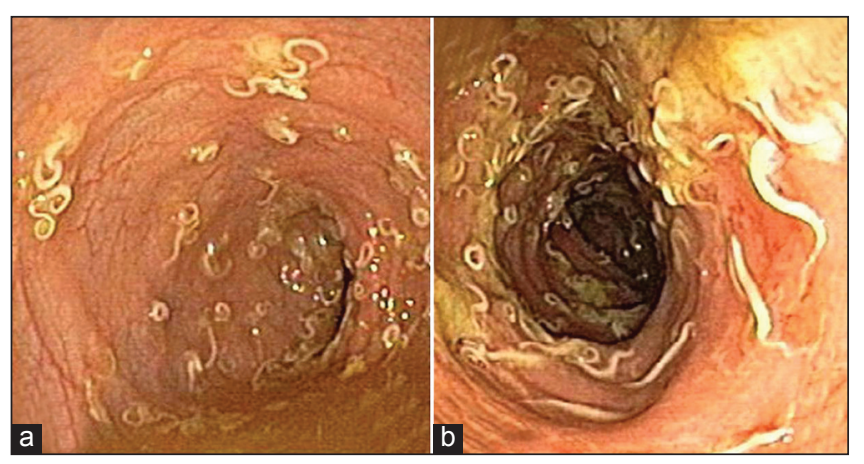

Figure 2: Colonoscopic picture of Extensive Trichuriasis involving the terminal ileum and the entire colon 
poor sanitation and unhygienic living conditions, history of pica could have made her more susceptible for reinfection.

In conclusion, extensive Trichuris trichura infection should be considered as a differential diagnosis in children who present with rectal prolapse and gastrointestinal bleed. Awareness about this condition among clinicians, regular follow up by patients, periodic deworming of the children and health education among the public may be useful to prevent this infection and its complications.

\section{ACKNOWLEDGMENT}

Department of Paediatrics, St. John's Medical College and Hospital. Bangalore

\section{REFERENCES}

1. Azira NM, Zeehaida M. Severe chronic Iron deficiency anemia secondary to Trichuris dysentary syndrome-A case report. Trop Biomed 2012;29: 626-31.

2. Tuan Sharif SE, Ewe Seng C, Mustaffa N, Mohd Shah NA, Mohamed Z. Chronic Trichuris trichura Infection Presenting as lleocaecal Valve Swelling Mimicking Malignany. ISRN Gastroenterol 2011;2011:105178.
3. Fatallah HI, Akbar HO. Trichris trichura induced massive lower gastrointestinal hemorrhage - A Rare Presentation. Internet J Gastroenterol 2010;9.

4. Lorenzetti R, Campo SM, Stella F, Hassan C, Zullo A, Morini S. An unusual endoscopic finding: Trichuris trichura Case report and review of literature. Dig Liver Dis 2003;35:811-3.

5. Diniz-Santos DR, Jambeiro J, Mascarenhas RR, Silva LR. Massive Trichuris trichura infection as a cause of chronic bloody diarrhoea in a child. J Trop Paediatr 2006;52:66-8.

6. Amal RN, Eugene CB, Yunus AM. Persistent trichuriasis in an Orang Asli child. Indian J Practicing Doctor 2008;5:4.

7. Stephensen LS, Holland CV, Cooper ES. The Public health significance of Trichuris trichura. Parasitology 2000;121(Suppl):S73-95.

8. Do KR, Cho YS, Kim HK, Hwang BH, Shin EJ, Jeong HB, et al. Intestinal Helminthic infection Diagnosed by Colonoscopy in a Regional Hospital during 2001-2008. Korean J Parasitol 2010;48:75-8.

9. Ok KS, Kim YS, Song JH, Lee JH, Ryu SH, Lee JH, et al. Trichuris trichura infection diagnosed by colonoscopy: Case reports and review of literature. Korean J Parasitol 2009;47:275-80.

How to cite this article: Gachinmath SB, Pratibha S, Dias M, Mallikarjun P. Extensive persistent Trichuriasis: A case report. Int J Med Public Health 2014;4:523-5.

Source of Support: Nil, Conflict of Interest: None declared. 